ORIGINAL STUDY

\title{
Correlations between morphology and ultrasound exam in cases with nasal and paranasal sinuses pathology
}

\author{
Mihai Dumitru', Daniel Tweedie ${ }^{2}$, Ion Anghel ${ }^{1,3}$, Romica Cergan $^{4}$, Codrut Sarafoleanu $^{1,5}$, \\ Adrian Costache ${ }^{6}$ \\ 1"Carol Davila” University of Medicine and Pharmacy, Bucharest, Romania \\ ${ }^{2}$ Guy's and St Thomas' NHS Foundation Trust, London, UK \\ ${ }^{3}$ ENT Department, Coltea Clinical Hospital, Bucharest, Romania \\ ${ }^{4}$ Anatomy Department, "Carol Davila” University of Medicine and Pharmacy, Bucharest, Romania \\ ${ }^{5}$ ENT\&HNS Department, "Sfanta Maria” Hospital, Bucharest, Romania \\ ${ }^{6}$ Ultrasound Teaching Center, “Carol Davila” University of Medicine and Pharmacy, Bucharest, \\ Romania
}

\begin{abstract}
BACKGROUND. Ultrasonography has been used in rhinology for diagnosing trauma lesions (fractures, hemosinus), second opinion in tumoral pathology, screening for sinusitis, but on a small scale and with future prospects of cost efficiency.

OBJECTIVE. We hope to grow awareness of the possible use of ultrasound in screening for nasal and paranasal sinuses pathology at the level of ENT emergency departments.

MATERIAL AND METHODS. We describe the technique for ultrasound examination of this region, emphasizing the need for a profound anatomical knowledge characteristic for ENT specialists. Any specialist having access to an ultrasound machine is encouraged to experiment with this imaging procedure. Two cases benefited from the use of ultrasonography in order to receive a better management and a swift treatment. One case presented with a maxillary sinus tumor and another with a paranasal tumor neighbouring the orbit.

CONCLUSION. Ultrasonography of nasal and paranasal sinuses permits serial examination without irradiating the patient; it could be implemented as an addition to FAST-like protocols at the level of emergency departments in order to screen for occult head and neck pathology prior to conventional radiology and CT imaging and thus reducing costs.
\end{abstract}

KEYWORDS: ultrasound, rhinology, nasal pathology, paranasal pathology

\section{INTRODUCTION}

One of the first references concerning the use of ultrasound in examining paranasal sinuses dates from $1972^{1}$. This was the era of A-mode ultrasonography which permitted the use of ultrasound mostly in the case of sinusitis, but this will improve with further development of devices and probes ${ }^{2}$. However, with the revolution in CT, ultrasound was forgotten and reemerged in emergency settings and in paediatric clinics. In emergency setting, ultrasonography of the sinuses can be an extension of FAST-like (focused abdominal sonogram for trauma) protocols in order to detect hemosinus or acute sinusitis as early as possible with a minimum use of resources ${ }^{3}$.

In paediatric clinics, ultrasonography could be used for a non-radiating detection of sinusitis, lowering the use of conventional radiology on children and enabling serial examination of the child during treat- 
ment $^{4,5}$. This principle can be applied also in the case of paediatric trauma with nasal bone fractures ${ }^{6}$. Numerous papers analyze the use of ultrasonography in nasal trauma and compare sensitivity and specificity with conventional radiology and $\mathrm{CT}^{7-10}$; these efforts lead to the work of Abu-Samra $\mathrm{M}$ et al. ${ }^{11}$ stating the role of intra-operative ultrasound-guided reduction of nasal bone fracture in patient satisfaction and patient nasal profile.

Two exotic uses of ultrasonography in rhinology are for anterior nasal fossa pathology and in the management of nasal allergen challenge $\mathrm{e}^{12-14}$.

\section{OBJECTIVE}

We present the technique for examining the nose and paranasal sinuses along with underlining the anatomical landmarks. A profound knowledge of paranasal sinus anatomy is the key to identifying subsequent pathology. Nowadays, the healthcare specialist works under pressure from a financial and time management point of view and the use of ultrasound in the region of the nose as an extension to FAST-like protocols in emergency settings may be an answer to this deadlock.

\section{MATERIAL AND METHODS}

We present two cases with nasal and paranasal sinuses pathology that received ultrasound examination. All cases had the ultrasonographic findings con- firmed by CT and underwent surgery with subsequent pathology diagnosis. All images presented in this paper come from patients admitted to the ENT Department, Coltea Clinical Hospital, Bucharest, Romania. All patients included signed an Informed Consent in compliance with the Declaration of Helsinki and current Good Clinical Practice.

For the examination of the patients a Sonoscape S2 machine with linear probe was used. The examination is performed with the patient seated in upright position, with the head flexed anteriorly to a 90-degree angle, so as to concentrate any fluid characteristic of sinusitis on the anterior wall of the sinus. The first step is a transverse view of the maxillary sinus; usually, the paranasal sinuses are empty air-filled cavities that present only an artefact like signature, called a posterior wall echo, resulted from the waves hitting the posterior wall of the sinus: PAS the anterior wall of the maxillary sinus, PPS - the posterior wall echo of the maxillary sinus (Figure 1).

The same imaging technique is applied in Figure 2, for examining the frontal sinus: $\mathrm{OF}$ - the posterior wall of the frontal sinus.

With an oblique lateral placement of the transducer along the bony nose, one can obtain a longitudinal view of the integrity of the bone frame, as it can be seen in Figure 3.

\section{Case 1}

We present the case of a 74-year-old male patient accusing headache and complete obstruction of the right nasal fossa. Routine nasal endoscopy found that the obstruction was caused by a tumor. The CT-scan revealed a

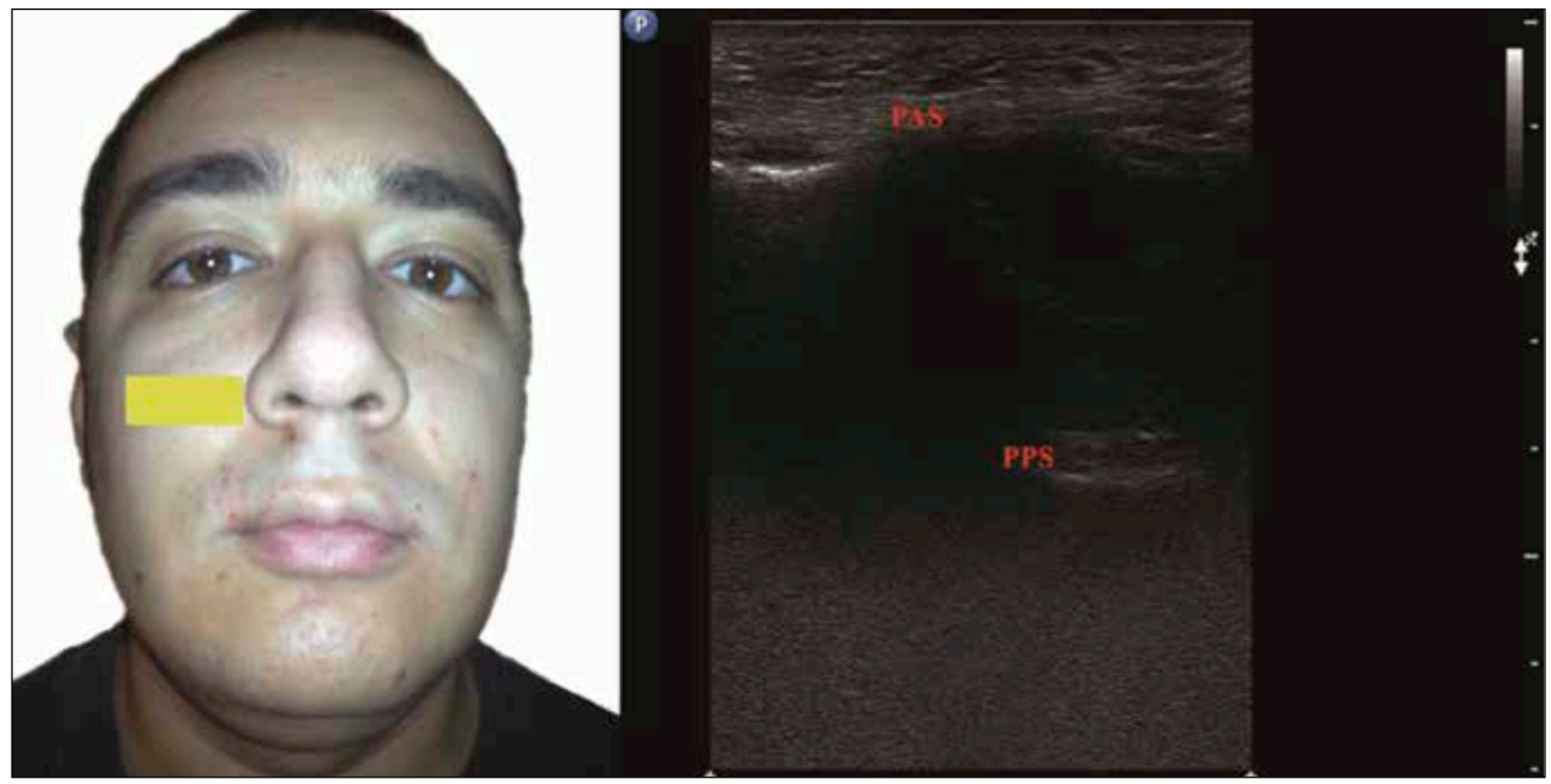

Figure 1 Normal ultrasonography of the maxillary sinus; PAS - anterior sinus wall, PPS - posterior sinus wall 


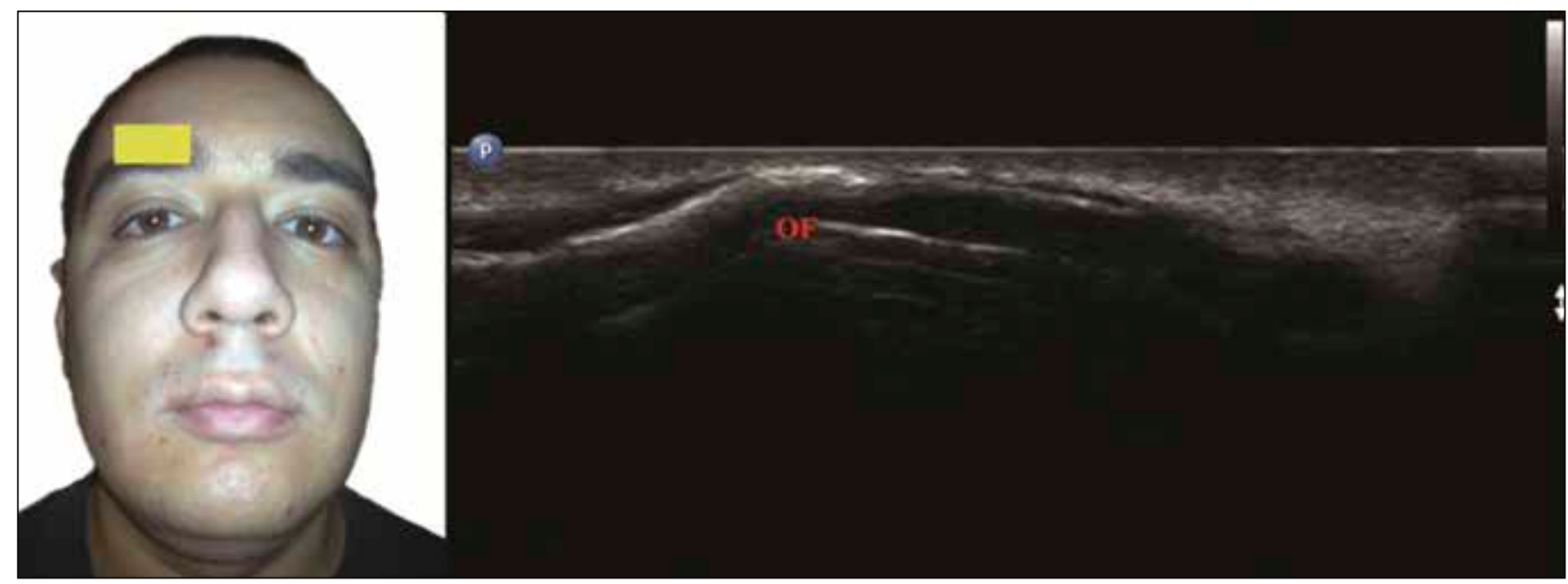

Figure 2 Transverse view of the frontal sinus; OF - frontal bone

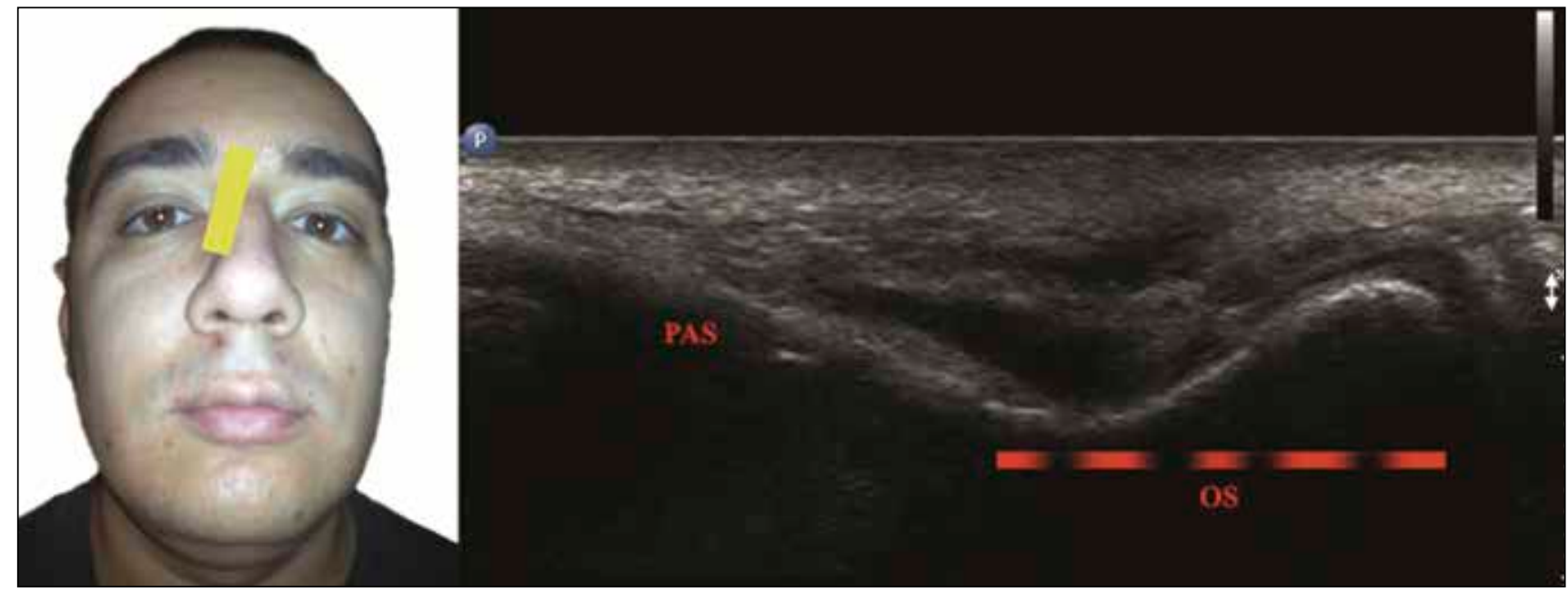

Figure 3 Longitudinal view of the nasal bones; PAS - frontal sinus anterior wall, OS - nasal bones

right maxillary sinus tumor of $43 / 39 \mathrm{~cm}$ which destroyed the medial wall of the sinus. Sonography revealed a contiguous propagation of the sound waves till the level of the posterior wall of the right maxillary sinus. Comparative ultrasound views of both maxillary sinuses dismissed the possibility of pansinusitis. Moreover, the dynamic ultrasound imaging performed while moving the patient's head at different angles showed that the content of the sinus was not varying in thickness; this implied the presence of a solid hypoechoic mass. In Figure 4 we illustrate the comparative view of the maxillary sinus on both sides correlated with the CT findings. Intraoperative findings and histopathologic results confirmed the diagnosis of an inverted papilloma.

\section{Case 2}

A 28-year-old patient referred to our ENT Department after undergoing successive ophthalmology con- sults, presenting a left paranasal tumor impairing the movement of the upper left eyelid. The cranio-facial CT-scan confirmed the integrity of the nasal bone structure and underlined the presence of a conjunctive tissue mass. The ultrasonography revealed a hypoechoic, poorly delineated oval mass on the left lateral part of the nose. In Figure 5 we present the ultrasound view of the tumor measuring 2.38 per $1.73 \mathrm{~cm}$ and the integrity of the nasal bony structure. Both CT and sonography excluded the contact with the eye ball, as the tumor only compressed adjacent structures. During surgery, the removed specimen confirmed our suspicion of a cartilaginous tumor. Unfortunately, immunohistochemistry analysis of the specimen is further needed in order to confirm the initial histopathologic diagnosis of desmoplastic cartilaginous tumor, probably derived from the cartilaginous structure of the nose. 


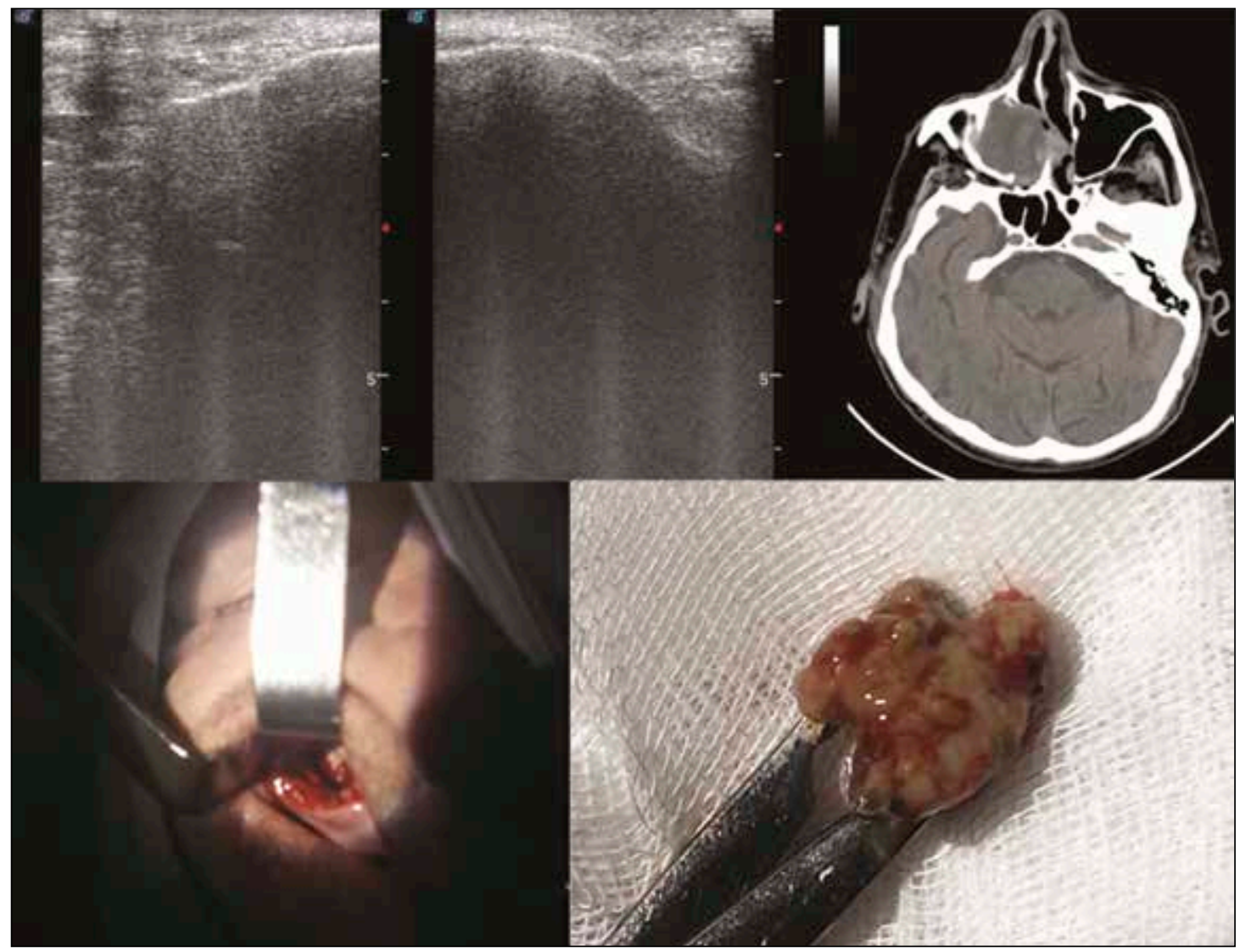

Figure 4 Case 1 - Ultrasonography view, CT and surgical aspect in a patient with sinus tumor: Upper left image - comparative ultrasound image of the right and left maxillary sinuses; Upper right image - CT section; Lower left image - classic surgical approach of the right maxillary sinus; Lower right image - part of the sinus content

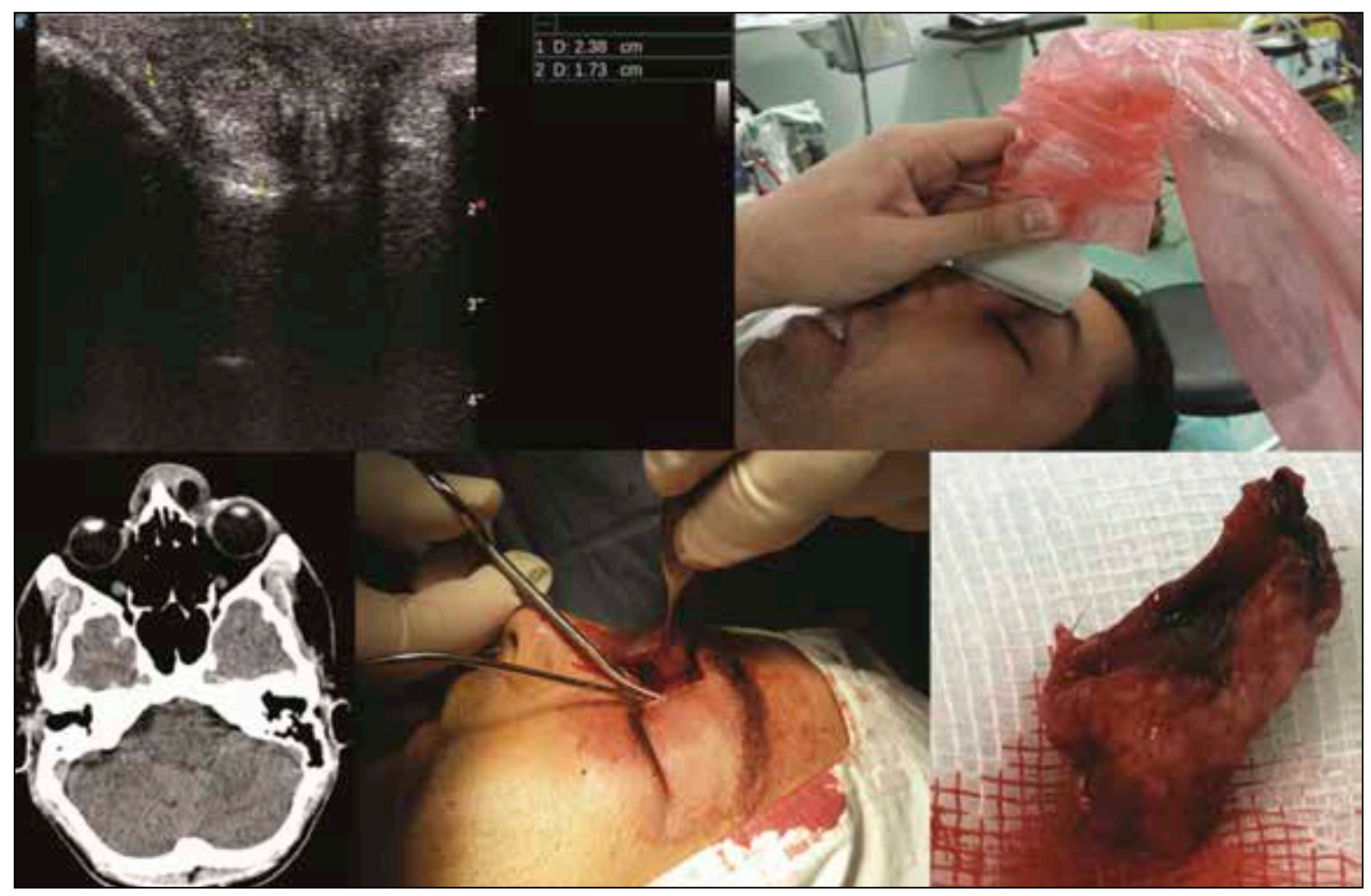

Figure 5 Case 2 - Ultrasonography view, CT and surgical aspect in a patient with paranasal tumor: Upper left image - ultrasound image of the mass; Upper right image - positioning of the ultrasound probe; Lower left image - CT slice; Lower middle image - intraoperatory aspect; Lower right image - macroscopic aspect of the mass removed 


\section{DISCUSSIONS}

The first documented use of ultrasound, in order to diagnose a rhinologic disease, comes from Russia in 1964 by Abdurasulov DM, et $\mathrm{al}^{15}$. They analyzed the use of ultrasound for diagnosing maxillary sinusitis.

Afterwards, till late 90's, there was a low constant rate of articles published on the subject of sonography and sinusitis, comparing this diagnostic imaging method first with standard plain radiography and later with CT. Evidently, with the revolution that computed tomography realized in the field of medicine, ultrasound dropped into shadow and oblivion when speaking about nasal pathology. CT and MRI are the gold standard for investigating a rhinologic patient.

The evolution of more powerful ultrasound transducers and devices revitalized the number of publications on this subject after 2010. One possible advantage of sonography is that it is fast and does not require the movement of the patient to specific imaging departments. Thus, some teams reuniting mainly maxillofacial surgeons analyzed the possibility of using ultrasound for trauma patients with promising results, but requiring experience with a greater number of cases. Another advantage of sonography is the lack of irradiation and this generated the possibility of using this investigation in children and pregnant women for confirmation of clinical diagnosis. But, even this aspect requires further study on a broader population.

There was even an attempt to use serial ultrasound examinations of mucosal thickness during allergy therapy with a major reduction in radiation dose. The most recent trend in research concerning the use of ultrasound imaging in rhinology analyzes the assessment of nasal fracture reduction at the end of the procedure and before, applying external contention devices that otherwise impair radiologic check-up.

However, there remains a major limitation for ultrasound in that one cannot plan an adequate functional endoscopic sinus surgery based only on ultrasonography exams.

\section{CONCLUSIONS}

Ultrasonography can be used as a screening tool for nasal and paranasal pathology. So, if you perform an ultrasound exam of the head and neck region, do not forget to have a look at the nose for incidental findings.

Ultrasonography could be used primarily in the case of children and pregnant women for confirma- tion of clinical findings and prior to advanced imaging using MRI.

In a busy intensive care unit where FAST (focused abdominal sonogram for trauma) protocol is routinely performed for trauma patients, use the same ultrasound machine to rule out nasal fractures or to discover hemosinus prior to confirmation by radiology.

Nevertheless, additional evidence-based medicine data is needed to confirm the place of this exotic imaging tool in the diagnostic management of nasal and paranasal pathology.

Acknowledgements: This paper is supported by the Sectoral Operational Programme Human Resources Development (SOP HRD), financed from the European Social Fund and by the Romanian Government under the contract number POSDRU/159/1.5/S/137390.

Conflict of interest: The authors have no conflict of interest.

\section{REFERENCES}

1. Heidelbach J.G., Gilbricht E. - Practical experience in ultrasonic diagnosis of the great paranasal sinuses and their bases. Z Arztl Fortbild (Jena)., 1972;66(4):208-10

2. van Duijn N.P., Brouwer H.J., Lamberts H. - Use of symptoms and signs to diagnose maxillary sinusitis in general practice: comparison with ultrasonography. BMJ, 1992;305(6855):684-687.

3. Bektas F., Soyuncu S., Yigit O. - Acute maxillary sinusitis detected by bedside emergency department ultrasonography. Int J Emerg Med, 2010;3(4):497498.

4. Pahakka T., Heikkinen T., Makela M.J., Alanen A., Kallio T., Korsoff L, Suonpaa J., Ruuskanen O. - Validity of ultrasonography in diagnosis of acute maxillary sinusitis. Arch Otolaryngol Head Neck Surg., 2000;126(12):14821486.

5. Fufezan O., Asavoaie C., Chereches Panta P., Mihut G., Bursasiu E., Anca I., Iacob D., Gocan H., Valean C. - The role of ultrasonography in the evaluation of maxillary sinusitis in pediatrics. Med Ultrason., 2010;12(1):4-11.

6. Hong H.S., Cha J.G., Paik S.H., Park S.J., Park J.S., Kim D.H., Lee H.K. - HighResolution Sonography for nasal fracture in children. AJR Am J Roentgenol., 2007;188(1):W86-92.

7. Thiede O., Kromer J.H., Rudack C., Stoll W., Osada N., Schmal F. Comparison of Ultrasonography and Conventional Radiography in the Diagnosis of Nasal Fractures. Arch Otolaryngol Head Neck Surg., 2005;131(5):434-439.

8. Lee M.H., Cha J.G., Hong H.S., Lee J.S., Park S.J., Paik S.H., Lee H.K. Comparison of High-Resolution Ultrasonography and Computed Tomography in the Diagnosis of Nasal Fractures. J Ultrasound Med., 2009;28(6):717-723

9. Adeyemo W.L., Akadiri O.A. - A systematic review of the diagnostic role of ultrasonography in maxillofacial fractures. Int J Oral Maxillofac Surg., 2011;40(7):655-661. doi: 10.1016/j.ijom.2011.02.001. Epub 2011 Mar 5.

10. Mohammadi A., Ghasemi-Rad M. - Nasal bone fracture - ultrasonography or computed tomography? Med Ultrason., 2011;13(4):292-295.

11. Abu-Samra M., Selmi G., Mansy H., Agha M. - Role of intra-operative ultrasound-guided reduction of nasal bone fracture in patient satisfaction and 
patient nasal profile (a randomized clinical trial). Eur Arch Otorhinolaryngol., 2011; 268(4):541-6. doi: 10.1007/s00405-010-1401-1. Epun 2011 Oct 21.

12. Pruna X., Inaraja L., Gallardo E., Serra J., Casamitjana F., Serrano A. - Value of Sonography in the Assessment of Space-Occupying Lesions of Anterior Nasal Fossa. J Clin Ultrasound., 2000;28(1):14-19.

13. Pelikan Z. - Diagnostic value of nasal allergen challenge combined with radiography and ultrasonography in chronic maxillary sinus disease. Arch
Otolaryngol Head Neck Surg., 2009;135(12):1246-1255. doi: 10.1001/archoto.2009.189.

14. Costache A., Dumitru M., Anghel I., Cergan R. - Atlas de anatomie ecografica in otorinolaringologie. SITECH, Craiova, 2013.

15. Abdurasulov D.M., Amilova A.A., Fazylov A.A., et al. - On the use of ultrasonics in the diagnosis of the maxillary sinuses. Nov Med Tekh., 1964:2:30-33. 